BI-TP 98/09

August 1998

revised version

\title{
Diquark Masses from Lattice QCD
}

\author{
M. Heß, F. Karsch, E. Laermann and I. Wetzorke \\ Fakultät für Physik, Universität Bielefeld, D-33615 Bielefeld, Germany
}

\begin{abstract}
We present first results for diquark correlation functions calculated in Landau gauge on the lattice. Masses have been extracted from the long distance behaviour of these correlation functions. We find that the ordering of diquark masses with spin 0 and 1 states in colour anti-triplet and sextet channels is in accordance with instanton motivated interaction models. Although we find evidence for an attractive interaction in colour anti-triplet states with a splitting between spin 0 and spin 1 diquarks that can account for the mass splitting between the nucleon and the delta, there is no evidence for a deeply bound diquark state.
\end{abstract}

\section{INTRODUCTION}

Since a long time it has been speculated that QCD at finite baryon number density and low temperature may have a much richer phase structure than at high temperature and low or vanishing baryon number density. In general this is due to the fact that quantum statistics is much more important at low temperature and energetically favours bosonic forms of matter over fermionic matter. At low temperature and low density the possibility of pion and kaon condensates thus has been discussed. Unlike in the high temperature case, where it is evident that the strongly interacting matter exists in the form of a quark-gluon plasma it has been speculated that at high baryon density bosonic states - diquarks and even larger quark clusters - may play an important 
role [1]. Recently it has been suggested that diquarks may, in fact, form a Bose condensate at high densities and low temperatures [2,3] and also the possibility of a dibaryon phase has again been discussed 沟.

The realization of such states of matter crucially depends on details of the interaction among quarks. In particular, the analysis of the fine structure of the hadron spectrum such as the nucleondelta mass splitting suggests that there exists a strong attractive force between quarks in a colour anti-triplet state with anti-parallel spin orientation which leads to the formation of a diquark state. Such a spin dependent interaction naturally arises in the framework of perturbative QCD from one-gluon exchange [5]. Furthermore it has been realized that a spin and flavour dependent interaction among constituent quarks is needed to account for a satisfactory description of the fine structure of the experimentally observed baryon spectrum [6]. Such an attractive spin and flavour dependent interaction indeed is induced by instantons [7,8]. This raises the possibility for the existence of rather tightly bound spin 0 diquark states. Some indications for such states have been found from the analysis of diquark correlation functions in the instanton liquid model [9]. A quantitative analysis of the interaction between quarks is clearly needed to judge the existence of the interesting diquark phase structure discussed in [2[3]. In particular, the possible coexistence of a chiral symmetry broken phase with a diquark condensate will crucially depend on the value of the diquark masses.

In this letter we will present first results from a calculation of diquark correlation functions on the lattice. In particular we present results for diquarks in the colour anti-triplet representation. Our spectrum calculations have been performed in quenched QCD using perturbatively improved gauge and fermion actions. As quark and diquark correlation functions are gauge-variant observables we have performed all our calculations in a fixed gauge 2 . We have used the Landau gauge as is commonly done in the analysis of quark [10,11] and gluon masses 12, 13, on the lattice.

\footnotetext{
${ }^{a}$ Alternatively one could couple the light diquark states to a heavy quark, which serves to neutralize the colour 9]. This, however, only trades gauge dependence against path dependence.
} 


\section{QUARK AND DIQUARK CORRELATION FUNCTIONS}

While it is generally expected that the interaction between up and down quarks in a spin 0 colour anti-triplet state is attractive, it is not that obvious how quarks in other quantum number channels interact. The analysis of diquark states in the instanton liquid model suggests a quite deeply bound spin 0 diquark state [9]. Such calculations, however, may miss a contribution from the confining part of the quark-quark interaction. In general the diquark masses will receive contributions from the constituent masses, the confining and the spin-dependent part of the quark-quark interaction. In terms of a simple potential model these contributions are to leading order additive [6],

$$
m_{F S C}=2 \bar{m}_{q}+V_{\text {conf }}+V_{S}
$$

where the subscript $F S C$ denotes flavour, spin and colour quantum numbers, $\bar{m}_{q}$ denotes the constituent quark mass, $V_{\text {conf }}$ and $V_{S}$ are the contributions from the confining and spin-dependent parts of the quark-quark potential. The latter may include contributions from flavour-spin as well as colour-spin dependent interaction terms. Some information on the contribution of the different terms to the diquark masses exists from the analysis of hadron masses within the framework of potential models. In the case of three quark states the corresponding form of Eq. II.1 reads, for instance, $m_{N}=3 \bar{m}_{q}+\tilde{V}_{\text {conf }}+2 V_{0}+V_{1}$ for the nucleon and $m_{\Delta}=3 \bar{m}_{q}+\tilde{V}_{\text {conf }}+3 V_{1}$ for the

delta, with $\tilde{V}_{\text {conf }}=3 V_{\text {conf }}$. While the masses are expected to receive the largest contributions from the constituent mass term $\left(\bar{m}_{q} \simeq 300 \mathrm{MeV}\right)$ and the confining part $\left(V_{\text {conf }} \simeq 200 \mathrm{MeV}\right.$ [6] $)$ the nucleon-delta mass splitting is entirely determined by the difference in the spin dependent part of the potential. The latter also is related to the mass splitting of the $S=0$ and $S=1$ diquarks,

$$
m_{613}-m_{303}=\frac{1}{2}\left(m_{\Delta}-m_{N}\right) .
$$

In the following we will analyze $S=0$ and $S=1$ diquark states in different colour and flavour

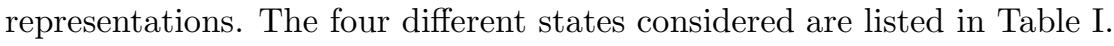

The corresponding diquark correlation functions are built up from the quark correlation function $G_{\alpha, \beta}^{a b}$. For the colour anti-triplets we obtain, for instance, 


\begin{tabular}{cccc}
\hline \hline$(F, S, C)$ & state & F-S coupling & C-S coupling \\
\hline$\left(3^{*}, 0,3^{*}\right)$ & $\epsilon_{a b c}\left(C \gamma_{5}\right)_{\alpha \beta} u_{a, \alpha}^{\dagger} d_{b, \beta}^{\dagger}$ & -2 & -2 \\
$\left(6,1,3^{*}\right)$ & $\epsilon_{a b c} u_{a, \alpha}^{\dagger} u_{b, \alpha}^{\dagger}$ & $-1 / 3$ & $2 / 3$ \\
$\left(3^{*}, 1,6\right)$ & $u_{c, \alpha}^{\dagger} d_{c, \alpha}^{\dagger}$ & $2 / 3$ & $-1 / 3$ \\
$(6,0,6)$ & $\left(C \gamma_{5}\right)_{\alpha \beta} u_{c, \alpha}^{\dagger} u_{c, \beta}^{\dagger}$ & 1 & 1 \\
\hline \hline
\end{tabular}

TABLE I. Diquark states with spin $S$ in flavour $(F)$ and colour $(C)$ anti-triplet and sextet representations. The third and fourth column give the relative strength of interaction terms corresponding to a flavour-spin and colour-spin coupling, $V_{S} \sim\left(\lambda_{1}^{a} \lambda_{2}^{a}\right)\left(s_{1} s_{2}\right)$, where $\lambda_{i}^{a}$ denote the generators of $S U(3)_{\text {flavour }}$ or $S U(3)_{\text {colour }}$, respectively.

$$
\begin{aligned}
& G_{c f}^{303}(\vec{x}, t)=\epsilon_{a b c} \epsilon_{d e f}\left(C \gamma_{5}\right)_{\alpha \beta}\left(C \gamma_{5}\right)_{\gamma \delta} G_{\alpha \gamma}^{a d} G_{\beta \delta}^{b e} \\
& G_{c f}^{613}(\vec{x}, t)=\epsilon_{a b c} \epsilon_{d e f}\left(G_{\alpha \beta}^{a d} G_{\alpha \beta}^{b e}-G_{\alpha \beta}^{a e} G_{\alpha \beta}^{b d}\right)
\end{aligned}
$$

where Latin (Greek) indices denote colour (spinor) degrees of freedom.

We have analyzed these correlation functions in Landau gauge. To be specific, we have calculated the diagonal correlators, $G^{F, S, C}(t) \equiv G_{a, a}^{F, S, C}$ and the scalar part of the quark propagator, $G_{q}(t) \equiv$ $4 G_{\alpha, \alpha}^{a, a}$ where the sum is taken over $a$ and $\alpha$ and also over the spatial coordinates $\vec{x}$ in order to project onto zero-momentum states.

Our calculations have been performed on lattices of size $16^{3} \times 32$. The gauge field configurations have been generated with a tree-level Symanzik improved action at a gauge coupling $6 / g^{2}=4.1$. A calculation of the string tension at this value of the coupling leads to $\sqrt{\sigma} a=0.3773(22)$, i.e. a cut-off $a^{-1} \simeq 1.1 \mathrm{GeV} 14{ }^{\mid}$. We have generated 73 gauge field separated by 100 sweeps of 4

\footnotetext{
${ }^{b}$ Here and in the following we use $\sqrt{\sigma}=420 \mathrm{MeV}$ to set the scale for all masses. Although $\sqrt{\sigma}$ is experimentally less well determined than a hadron mass it is more appropriate to use it to set the scale in a quenched calculation where hadron masses are calculated for various values of the quark mass
} 
overrelaxation and 1 heat bath steps each. These are fixed to Landau gauge using an algorithm based on Fourier accelerated overrelaxation [15]. In the fermion sector we use the SheikholeslamiWohlert action with a tree-level clover coefficient [16. On each gauge field configuration the fermion matrix has been inverted four times, i.e. with source vectors at four different lattice sites, for eight different quark mass values. Our analysis thus is based on a sample of 292 quark propagators. Fits have been performed with one and two exponential functions. Leaving out successively data points at small time separations we look for stable results for the fitted masses. Typical results obtained from single exponential fits of quark, diquark and nucleon correlation functions are shown in Figure f Fits with two exponential functions do reach a plateau earlier and yield consistent results.

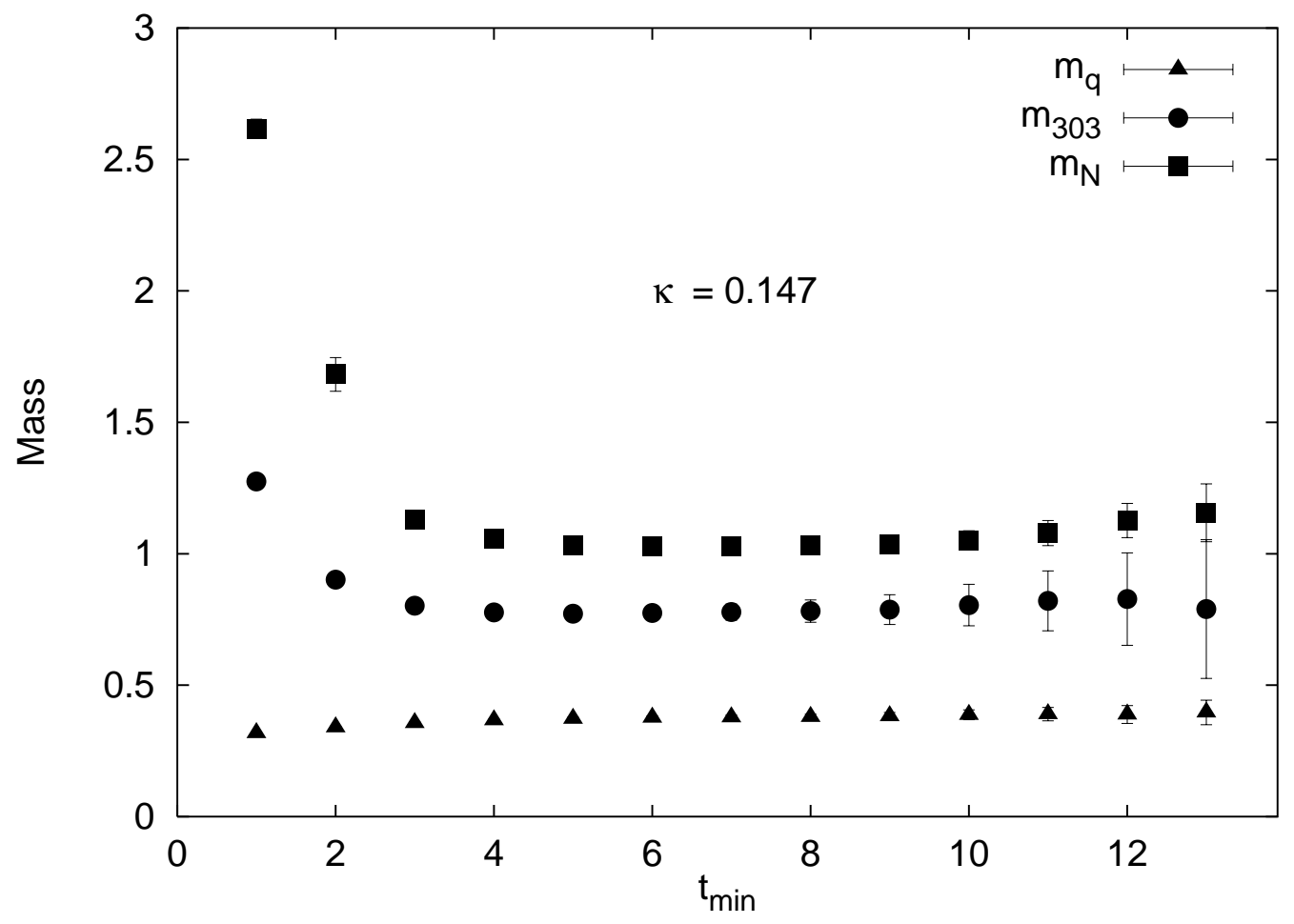

FIG. 1. Masses extracted from single exponential fits to the correlation functions of quark, colour anti-triplet spin 0 diquark and nucleon at $\kappa=0.147$ in the interval $\left[t_{\min }, 32-t_{\min }\right]$. 
In addition to the gauge dependent quark and diquark correlation functions we also construct the standard hadron correlation functions for the pion, rho, nucleon and delta. The 8 different quark masses selected correspond to the interval $0.5<m_{\pi} / m_{\rho}<0.9$. Our current analysis thus is still restricted to rather heavy quark masses. This is also reflected in the fact that the ratio $m_{N} / m_{\rho}$ is still close to that of the additive quark model, i.e. $m_{N} / m_{\rho} \simeq 1.5$. Nonetheless we note that our analysis does yield quite sizeable results for the nucleon-delta mass splitting (Table II) which was determined from a simultaneous fit of the ratio of nucleon and delta correlation functions and the nucleon correlation functions. From an extrapolation to the chiral limit we obtain $m_{\Delta}-m_{N}=184(63) \mathrm{MeV}$, which is about $40 \%$ below the experimental value. It is, however, consistent with earlier calculations with similar parameters [17, 18]. For our current analysis it is reassuring that we can observe a sizeable splitting in baryonic states and are thus sensitive to the spin dependence of hadron masses.

\begin{tabular}{lllll}
\hline \hline$\kappa$ & $m_{\pi}$ & $m_{\rho}$ & $m_{N}$ & $m_{\Delta}-m_{N}$ \\
\hline 0.140 & $0.910(1)$ & $1.025(2)$ & $1.608(6)$ & $0.065(10)$ \\
0.142 & $0.794(1)$ & $0.932(2)$ & $1.457(6)$ & $0.079(12)$ \\
0.144 & $0.667(1)$ & $0.836(3)$ & $1.298(6)$ & $0.098(16)$ \\
0.145 & $0.596(1)$ & $0.787(4)$ & $1.212(7)$ & $0.114(20)$ \\
0.146 & $0.519(1)$ & $0.739(7)$ & $1.122(10)$ & $0.128(22)$ \\
0.147 & $0.430(2)$ & $0.688(15)$ & $1.029(13)$ & $0.135(41)$ \\
0.1475 & $0.379(2)$ & $0.661(18)$ & $0.982(13)$ & $0.131(66)$ \\
0.148 & $0.316(3)$ & $0.595(66)$ & $0.924(14)$ & $0.147(97)$ \\
\hline$\kappa_{c}$ & & $0.579(18)$ & $0.821(13)$ & $0.166(56)$ \\
\hline \hline
\end{tabular}

TABLE II. Hadron masses and the nucleon-delta mass splitting for various values of the hopping parameter. The last row gives the results of an extrapolation to the chiral limit obtained from a linear fit in $\kappa^{-1}-\kappa_{c}^{-1}$. The critical value of the hopping parameter for vanishing pion mass has been determined as $\kappa_{c}=0.14923(2)$ 
The basic component for the analysis of diquark correlation functions is the quark propagator. Although the quark mass is, in principle, a gauge-variant quantity, it has been found to show only little gauge dependence in a class of covariant gauges, which includes the Landau gauge 10$]$. The quark masses extracted in Landau gauge are consistent with constituent quark mass values of $(350-400) \mathrm{MeV}$ [10,11]. Our results for the quark correlation function are consistent with these earlier findings. We generally observe that local masses extracted from $G_{q}(t)$ rise with increasing temporal distance $t$ and develop a plateau for $t \gtrsim 6$. In this region we have performed exponential fits to extract the quark mass. The results are summarized in the second column of Table III. Using the five largest $\kappa$-values to extrapolate linearly in $\kappa^{-1}-\kappa_{c}^{-1}$ to the chiral limit, we find $\bar{m}_{q} / \sqrt{\sigma}=0.813(30)$ or $\bar{m}_{q} \simeq 342(13) \mathrm{MeV}$.

\begin{tabular}{llll}
\hline \hline$\kappa$ & $\bar{m}_{q}$ & $m_{303}$ & $m_{613}$ \\
\hline 0.140 & $0.586(5)$ & $1.190(9)$ & $1.207(11)$ \\
0.142 & $0.531(5)$ & $1.079(12)$ & $1.102(13)$ \\
0.144 & $0.472(6)$ & $0.962(14)$ & $0.993(14)$ \\
0.145 & $0.442(6)$ & $0.901(15)$ & $0.936(15)$ \\
0.146 & $0.410(8)$ & $0.839(15)$ & $0.880(26)$ \\
0.147 & $0.378(11)$ & $0.774(15)$ & $0.827(33)$ \\
0.1475 & $0.361(12)$ & $0.737(18)$ & $0.806(35)$ \\
0.148 & $0.344(11)$ & $0.696(18)$ & $0.806(45)$ \\
\hline$\kappa_{c}$ & $0.307(11)$ & $0.623(19)$ & $0.727(37)$ \\
\hline \hline
\end{tabular}

TABLE III. Quark and diquark masses for various values of the hopping parameter. The last row gives the results of an extrapolation to the chiral limit obtained from a linear fit in $\kappa^{-1}-\kappa_{c}^{-1}$. 
The diquark correlation functions corresponding to the colour anti-triplet representation show a remarkably clean exponential decay. This is reflected in the small $t$-dependence of local masses shown in Figure 1 and also suggests the existence of an attractive interaction in this channel. Although the diquark correlation functions are also gauge variant the local masses show a behaviour very similar to that of ordinary hadron masses. They approach a plateau from above. In the case of the spin 0 diquark state this is typically reached already for $t \gtrsim 4$. For the spin 1 correlation functions we observe larger contributions from excited states at short distances and consequently the plateau is reached only for $t \gtrsim 6$. The situation is improved when we perform fits with two exponentials. These yield stable results for distances $t \gtrsim 2$. The mass values obtained from such fits are given in Table 1 and III. In Figure 2 we show results for half the spin 0 diquark masses and a combination of nucleon and delta masses, $\left(m_{\Delta}+m_{N}\right) / 6$, which generally is used as a definition of the constituent quark mass. As expected from potential models $m_{N} / 3$ is significantly lighter than the quark masses calculated by us in Landau gauge. We also note that in the chiral limit $\left(\kappa \equiv \kappa_{c}\right)$ the quark mass agrees quite well with $\left(m_{\Delta}+m_{N}\right) / 6$. This is quite astonishing, as this phenomenologically defined constituent quark mass receives, at least in the context of potential models, additional contributions from the confining part of the potentials as well as the spindependent parts. This difference also is reflected in the different hopping parameter (bare quark mass) dependence seen in Figure 2. 


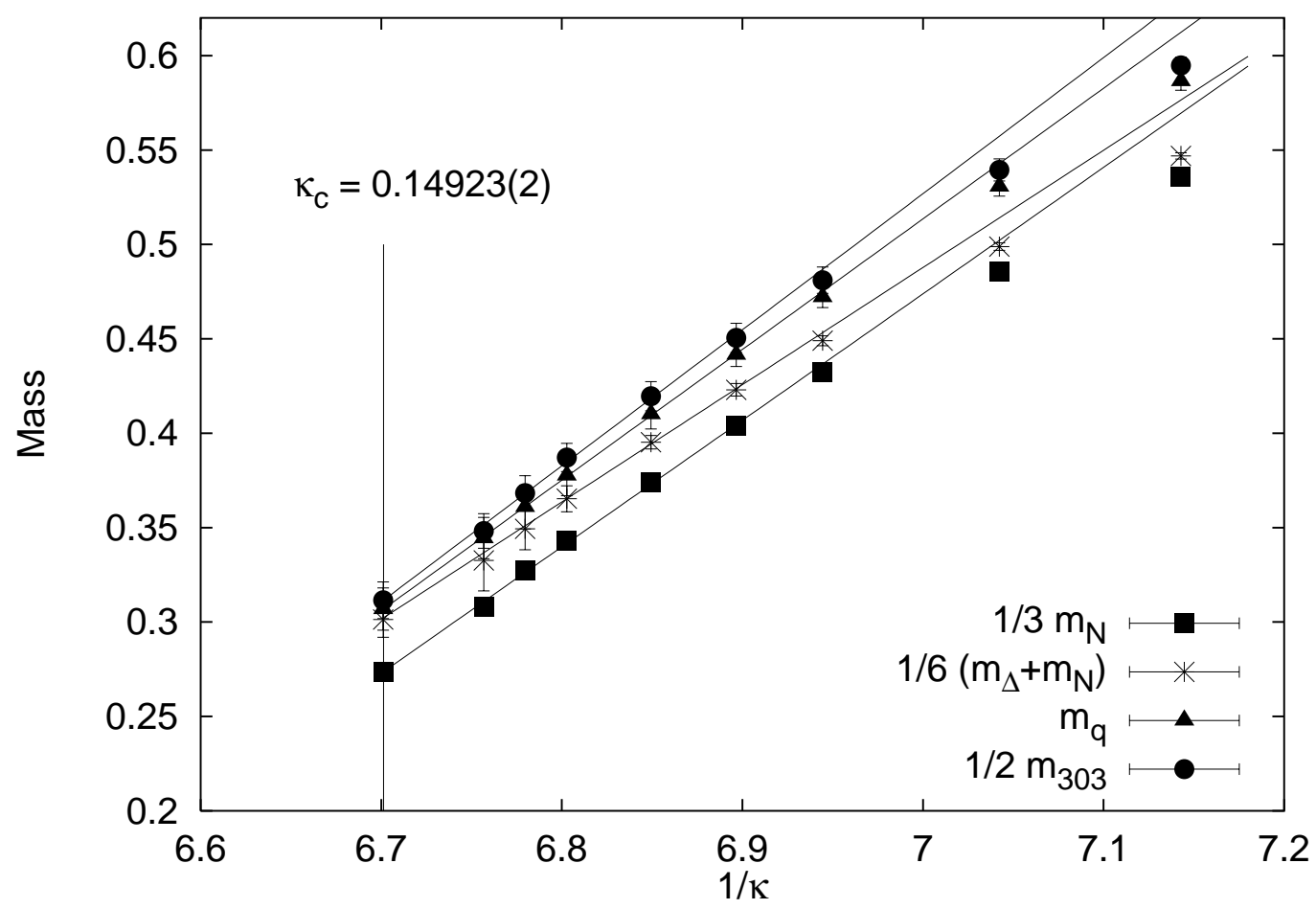

FIG. 2. 1/2 diquark(303), 1/3 nucleon and 1/6 (nucleon+delta) in comparison with the quark mass calculated in Landau gauge.

The mass of the spin 0 , colour anti-triplet diquark is slightly larger than twice the constituent quark mass. Interpreted again in terms of a potential model this suggests that the positive energy contribution resulting from confinement is just balanced by a contribution from an attractive spin interaction. From Figure 2 as well as Table II and III it is, however, obvious that the diquark masses show no indication for a deeply bound diquark state. In the tables we also give the result of an extrapolation to the chiral limit which is based on the five lightest quark masses and is obtained from a fit linear in $\kappa^{-1}-\kappa_{c}^{-1}$. Using again the string tension to set the scale we find $m_{303}=694(22) \mathrm{MeV}$.

Similar to what has been observed in the case of the delta-nucleon mass splitting we find that the difference between the masses of the spin 0 and spin 1 diquarks increases with decreasing quark mass. This is shown in Figure 3. From an extrapolation to the chiral limit we find $m_{613}-m_{303}=$ $0.104(42)$, which in fact is about $60 \%$ of the splitting found in the nucleon channel. This agrees 
well with the behaviour expected from potential models (Eq. II.2).

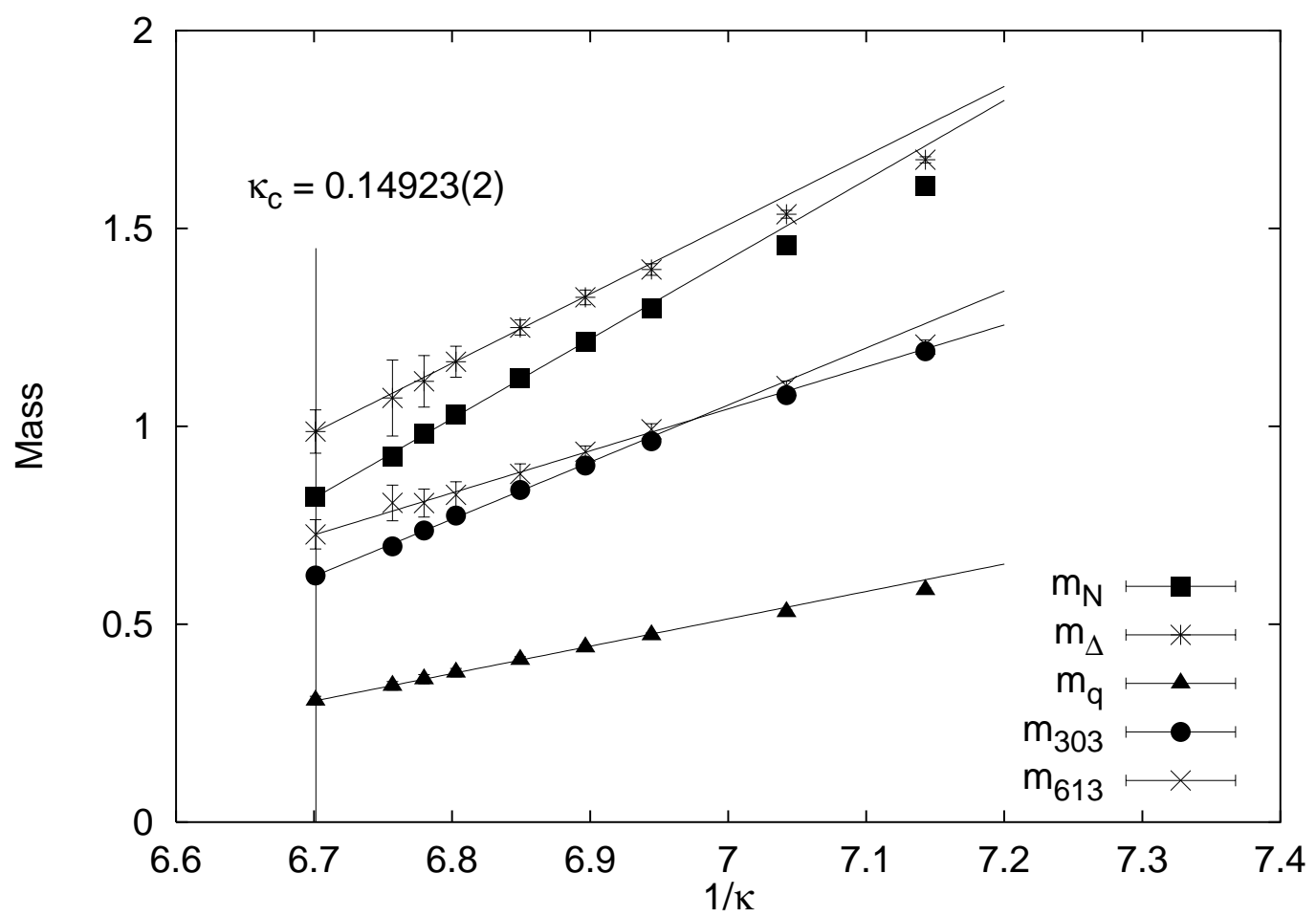

FIG. 3. Mass splitting between colour anti-triplet diquarks and nucleon/delta: The lines show fits to the results obtained with the five lightest quark masses.

Let us finally comment on the correlation functions for diquarks in a colour sextet representation. We find that these have a much smaller amplitude and are therefore much more noisy. Moreover, they receive much larger contributions from excited states at short distances and reach a plateau only very late. The general tendency is that the colour-sextet states have larger masses than the triplet states. This is in accordance with expectations based on a flavour-spin dependent coupling (Table [1). We will report on a more detailed analysis of these correlation functions elsewhere.

\section{CONCLUSION}

We have analyzed colour anti-triplet diquark states with spin 0 and 1 as well as quark masses in Landau gauge. We find that the mass splitting between spin 0 and spin 1 diquark states can 
account for the observed mass splitting between the nucleon and delta.

Although the current analysis has been performed in the quenched approximation of QCD on quite coarse lattices and with fairly large quark masses $\left(m_{\pi} / m_{\rho} \gtrsim 0.5\right)$ the current analysis does seem to rule out a deeply bound diquark state. For the lightest spin 0 state we find $m_{303} \simeq 700$ $\mathrm{MeV}$ which is slightly larger than twice the constituent quark mass.

This makes the existence of a diquark phase coexisting with a chiral symmetry broken phase as suggested in 2 unlikely. However, our finding of a sizeable splitting between the spin 0 and 1 antitriplet diquark states and the clear exponential decay of the corresponding correlation functions gives some support for an attractive q-q interaction in the spin 0 channel and for the existence of a colour superconducting diquark phase at high density. We should stress that our current analysis has been performed in the $T=0$ confining phase of QCD where the attractive interaction due to instantons (flavour-spin coupling) is expected to give the dominant contribution. In the high density regime it is expected that the instanton induced interactions become suppressed and one-gluon exchange (colour-spin coupling) becomes increasingly important for the attractive interaction. Further studies of the temperature and density dependence of diquark masses as well as the nucleon-delta splitting are thus needed. The latter will be difficult to analyze for 3-colour QCD due to the well-known algorithmic problems in QCD with non-vanishing chemical potential. The density dependence of the q-q interactions may, however, first be analyzed in 2-colour QCD [2].

\section{ACKNOWLEDGEMENTS}

This work was partly supported by the TMR network Finite Temperature Phase Transitions in Particle Physics, EU contract no. ERBFMRX-CT97-0122. The work of F.K. was partly supported by a NATO Collaborative Research Grant, contract no. 940451. 
[1] J.F. Donoghue and K.S. Sateesh, Phys. Rev. D38 (1988) 360.

[2] R. Rapp, T. Schäfer, E.V. Shuryak and M. Velkovsky, Phys. Rev. Lett. 81 (1998) 53.

[3] M. Alford, K. Rajagopal and F. Wilczek, Phys. Lett. B422 (1998) 247.

[4] A. Faessler, A.J. Buchmann, M.I. Krivoruchenko and B.V. Martemyanov, Phys. Lett. B391 (1997) 255 and J. Phys. G24 (1998) 791.

[5] A. de Rujula, H. Georgi and S.L. Glashow, Phys. Rev. D12 (1975) 147.

[6] see for instance: L. Ya. Glozman and D.O. Riska, Phys. Rep. 268 (1996) 263.

[7] G.'t Hooft, Phys. Rev. D14 (1976) 3432.

[8] E.V. Shuryak and J.L. Rosner, Phys. Lett. B218 (1989) 72.

[9] T. Schäfer, E.V. Shuryak and J.J.M. Verbaarschot, Nucl Phys. B412 (1994) 143.

[10] C. Bernard, D. Murphy, A. Soni and K. Yee, Nucl. Phys. B (Proc. Suppl.) 17 (1990) 593.

[11] J. I. Skullerud (UKQCD Collaboration), Nucl. Phys. B (Proc. Suppl.) 42 (1995) 364.

[12] J.E. Mandula and M. Ogilvie, Phys. Lett. 201B (1988) 117 and B248 (1990) 156.

[13] U.M. Heller, F. Karsch and J. Rank, Phys. Lett. B355 (1995) 511.

[14] B. Beinlich, F. Karsch, E. Laermann and A. Peikert, String Tension and Thermodynamics with Tree Level and Tadpole Improved Actions, hep-lat/9707023v2, to be published in EPJ C (The European Physical Journal C).

[15] C.T.H. Davies, G.G. Batrouni, G.R. Katz, A.S. Kronfeld, G.P. Lepage, K.G. Wilson, P. Rossi and B. Svetitsky, Phys. Rev. D37 (1988) 1581.

[16] B. Sheikholeslami and R. Wohlert, Nucl. Phys. B259 (1985) 572

[17] T. A. DeGrand, Nucl. Phys. B (Proc. Suppl.) 20 (1991) 353

[18] D. Weingarten, Nucl. Phys. B (Proc. Suppl.) 34 (1994) 29 